\title{
La energía y su impacto socioambiental en la prensa digital: temáticas y potencialidades didácticas para una educación CTS
}

\author{
Isabel Guerrero-Márquez iD \\ Universidad de Sevilla. España.isaguerrrero@gmail.com \\ Antonio García-Carmona iD \\ Departamento de Didáctica de las Ciencias Experimentales y Sociales. Universidad de Sevilla. España. \\ garcia-carmona@us.es
}

[Recibido: 31 enero 2020. Revisado: 2 mayo 2020. Aceptado: 5 mayo 2020]

\begin{abstract}
Resumen: La producción y consumo de energía es un asunto socio-científico de gran trascendencia en la actualidad. Constituye uno de los indicadores principales de progreso y bienestar social. El impacto económico, social y ambiental de las distintas fuentes de energía, los hábitos de consumo energético y su coste, o la determinación de estrategias para lograr un desarrollo energético sostenible son, entre otros aspectos, objeto de importantes debates. Por ello, el análisis de las interacciones Ciencia-Tecnología-Sociedad (CTS) ligadas a la energía debería ser uno de los objetivos clave de la educación científica básica. En este sentido, el presente artículo destaca el interés educativo que puede tener la lectura crítica y reflexiva de noticias publicadas en la prensa digital sobre este tópico. Asimismo, con el fin de valorar el potencial didáctico de este recurso, se hace un análisis sistemático de las noticias relacionadas con la energía, que fueron publicadas durante el periodo 20132018 en los principales diarios digitales españoles. Tras la aplicación de varios filtros de análisis, se selecciona una muestra final de 20 noticias cuyo contenido es examinado descriptivamente. Además, se hacen sugerencias didácticas concretas para implementar cada una de esas noticias en clase de ciencias. Estas sugerencias se concretan en posibles preguntas abiertas, y de cariz reflexivo, que pueden contribuir al desarrollo del pensamiento crítico y actitudes de responsabilidad en el alumnado ante asuntos CTS relacionados con la energía.
\end{abstract}

Palabras clave: alfabetización científica; educación científica; energía; noticias científicas; prensa digital; relaciones CTS.

Energy and its socio-environmental impact in the digital press: issues and didactic potentialities for STS education

\begin{abstract}
Energy production and consumption is currently considered a socio-scientific issue of enormous importance. It represents one of the main indicators of progress and social welfare. The economic, social and environmental impact of the different energy sources, the consumption habits of energy, the energy cost, among other aspects, are subject of relevant discussions. Thus, the analysis of Science-Technology-Society (STS) interactions related to energy should be a key goal for basic science education. In this sense, the present article highlights the educational interest of reading, in a critical a reflective manner, news of digital press related to that topic. Likewise, in order to value the didactic potential of such resource, a systematic analysis of news related to energy, which are published in the 2013-2018 period in the most important digital newspapers in Spain, is made. After applying various analysis filters, a final sample of 20 press news was selected whose content is analyzed descriptively. In addition, specific didactic suggestions for implementing each press news in science classroom are offered. These suggestions are concreted in asking open-ended and reflective questions, which can help students to develop both critical thinking abilities and responsibility attitudes regarding CTS issues related to energy.
\end{abstract}

Keywords: digital press; energy; science education; scientific literacy; scientific news; STS relationships.

Para citar este artículo: Guerrero-Márquez, I. y García-Carmona, A. (2020) La energía y su impacto socioambiental en la prensa digital: temáticas y potencialidades didácticas para una educación CTS. Revista Eureka sobre Enseñanza y Divulgación de las Ciencias 17(3), 3301. 10.25267/Rev_Eureka_ensen_divulg_cienc.2020.v17.i3.3301

\section{Alfabetización científica en asuntos CTS ligados a la energía}

En las últimas décadas, los avances científicos y tecnológicos han sido espectaculares, en tanto que han transformado nuestro estilo de vida y la sociedad, en general. Tal es así que es casi 
imposible discutir sobre temas políticos, económicos, sociales, ambientales o educativos sin recurrir al papel que la ciencia y la tecnología juegan en estos (Hodson 2003). Por ello, es necesario promover una alfabetización científica apropiada para que la ciudadanía afronte, de manera responsable, los retos y problemáticas que surgen en torno al desarrollo científicotecnológico (Hazelkorn et al. 2015).

Sin embargo, durante la última década, las vocaciones científicas del alumnado han disminuido considerablemente en Europa (Mejlgaard, Bloch, Degn, Nielsen y Ravn 2012; Rocard et al. 2007). Esta situación responde a múltiples causas (Solbes 2011), pero quizás una de las más destacables derive de una educación científica enfocada a promover una ciencia escolar desconectada del mundo en el que vivimos (Aragón 2004). Desde hace años, la investigación didáctica está indicando que la comprensión de ideas científicas y la adquisición de actitudes positivas hacia la ciencia se ven favorecidas si la enseñanza de las ciencias se plantea en clara conexión con la realidad cotidiana del alumnado (Bennett, Lubben y Hogarth 2007). En esa contextualización debería prestarse especial atención a las relaciones Ciencia-TecnologíaSociedad (CTS), dado que proveen escenarios idóneos para conocer los temas científicotecnológicos contemporáneos, entender su transcendencia social y, sobre todo, tomar conciencia de la necesaria implicación de la ciudadana ante ellos (Acevedo 2009; GarcíaCarmona 2005, 2006, 2008). Como argumenta Fleming (1986), la reflexión sobre los asuntos socio-científicos favorece en el alumnado una cognición social, que difícilmente se logra mediante una educación científica centrada solo en contenidos disciplinares.

Un asunto socio-científico de gran trascendencia, en la actualidad, es el relacionado con la producción y consumo de energía. No en vano, ello constituye uno de los indicadores principales de progreso y bienestar social (Gómez Romero 2007). La atención recurrente a temas relacionados con la energía en los medios de comunicación es también un claro indicador de su relevancia social. El impacto económico, social y ambiental de las distintas fuentes de energía, así como los ámbitos de consumo energético y su coste, o la determinación de estrategias para lograr un desarrollo energético sostenible, son objeto de importantes debates en el presente (García-Carmona y Criado 2008; Rose y Calabrese 2012). Por tanto, el análisis de las interacciones CTS ligadas a la energía debería ser considerado uno de los objetivos clave de la educación científica básica (Doménech et al. 2007; Solbes y Tarín 2004). Y ello, en efecto, se establece en el currículo nacional de ciencias (física y química) para Educación Secundaria Obligatoria (ESO, 12-16 años) en España (Real Decreto 1105/2014, 2015), mediante estándares de aprendizaje como los siguientes (p. 262):

- Reconoce, describe y compara las fuentes renovables y no renovables de energía, analizando con sentido crítico su impacto medioambiental.

- Compara las principales fuentes de energía de consumo bumano, a partir de la distribución geográfica de sus recursos y los efectos medioambientales.

- Analiza la predominancia de las fuentes de energía convencionales frente a las alternativas, argumentando los motivos por los que estas últimas aún no están suficientemente explotadas.

- Interpreta datos comparativos sobre la evolución del consumo de energía mundial proponiendo medidas que pueden contribuir al ahorro individual y colectivo.

La atención a estos aspectos se justifica, además, por que los estudiantes de ESO suelen desconocer las relaciones CTS vinculadas a la energía (Solbes y Tarín 2004). Demeo, Feldman y Peterson (2013) señalan, en este sentido, que gran parte de la ciudadanía no es capaz de establecer, por ejemplo, una conexión tangible entre el uso de combustibles fósiles y la degradación ambiental; lo cual les limita para mantener discusiones sensatas sobre nuestro futuro climático y energético. Esto es, en parte, consecuencia de un tratamiento educativo de la energía exclusivamente conceptual y descontextualizado (Nordine, Krajcik y Fortus 2011). Por tanto, la enseñanza de la energía desde una perspectiva CTS puede ser una posible vía 
didáctica, ya que no solo contribuye a mejorar la comprensión de los conceptos científicos de la energía, sino también el desarrollo de habilidades de pensamiento crítico y la toma de decisiones responsables ante asuntos socio-científicos relacionados con esta (García-Carmona y Criado 2010; Yuenyong, Jones, Sung-Ong, Yutakom y Forret 2006).

\section{Las noticias de la prensa digital como recurso para la educación CTS}

Los medios de comunicación constituyen una de las principales fuentes de información científica para la sociedad, y son grandes generadores de opinión pública sobre cuestiones socio-científicas (Jarman y McClune 2007). Especialmente la prensa diaria, y muy en particular su versión digital (FECYT 2018), contribuye a que una parte importante de la ciudadanía continúe desarrollando su alfabetización científica tras su paso por la educación formal (Hodson 2008; Miller 2004). Muchos diarios contienen una sección dedicada a temas científicos y tecnológicos actuales; aunque con frecuencia incluyen noticias relacionadas con la ciencia y la tecnología en otras secciones dedicadas a la política, la economía, la cultura, etc. Por tanto, la lectura regular de noticias científicas en clase de ciencias puede ayudar a que el alumnado comprenda y se posicione, con sentido crítico y responsable, ante los asuntos científicos y socio-científicos que en ellas se difunden (Jiménez-Liso, Hernández y Lapentia 2010; Oliveras y Sanmartí 2009). Asimismo, en un sentido más global, puede contribuir a que el alumnado tome consciencia del dinamismo permanente de la ciencia y la tecnología dentro del contexto sociocultural de cada época (García-Carmona 2014; García-Carmona y Acevedo 2016). En general, como señalan Hobbs y Jensen (2009), el uso de noticias científicas en la enseñanza de las ciencias puede proporcionar al alumnado experiencias educativas más auténticas.

No obstante, el empleo de noticias de la prensa como recurso en la educación científica requiere de un análisis previo sobre su pertinencia y potencial didáctico. En ocasiones, el contenido de las noticias está sesgado por la interpretación y/o intencionalidad periodística, así como por las posibles simplificaciones o falta de rigor en su exposición al pretender que cualquier lector o lectora comprenda el mensaje, con independencia de su formación científica (García-Carmona 2015). Si bien, el análisis de estos aspectos puede ser igualmente interesante, dentro de un enfoque contextualizado de la educación científica; sobre todo, si está encaminada al desarrollo de un pensamiento crítico y de actitudes precautorias en el alumnado. En tal perspectiva, la lectura crítica de noticias científicas no debe limitarse a comprender las palabras y almacenar el mensaje, sino que debe propiciar un proceso de construcción de significados teniendo en cuenta la intención y/o grado de credibilidad de la autoría (Oliveras, Márquez y Sanmartí 2013). En particular, debe favorecer la elaboración de opiniones y argumentos propios, con vistas a defender una posición o la toma de decisiones personales y sociales en torno a los asuntos tratados. Para ello, es preciso también que el alumnado active y revise sus ideas iniciales sobre ellos (Alfaro 2011).

\section{Objetivos}

De acuerdo con todo lo anterior, los objetivos del presente trabajo son los siguientes:

1. Analizar, de manera sistemática y descriptiva, la presencia y el contenido de noticias relacionadas con la energía, publicadas en las versiones digitales de los principales diarios españoles durante el periodo 2013-2018.

2. Sugerir posibles usos escolares de las noticias analizadas para el desarrollo de un pensamiento crítico y responsable del alumnado en torno a asuntos CTS relacionados con la energía, mediante el planteamiento de cuestiones para su reflexión en el aula. 


\section{Metodología}

En el marco de un análisis exploratorio y descriptivo, para abordar los objetivos se llevó a cabo una búsqueda bibliográfica en la prensa digital española, delimitada en el periodo de tiempo comprendido entre 2013 y el segundo trimestre de 2018. Los diarios españoles seleccionados para esa búsqueda fueron El País, El Mundo y $A B C$. La selección de estos diarios se justifica, en primer lugar, porque se encuentran entre los medios de comunicación de mayor tradición, difusión e influencia mediática en España durante las últimas décadas; y, en segundo lugar, porque incluyen una sección específica dedicada a la ciencia y tecnología.

La selección de la muestra de noticias analizada se hizo siguiendo las tres fases siguientes:

- Fase I. Entre los diarios seleccionados, se hizo una búsqueda por el tópico energía. Debido al gran número de resultados (más de 20.000 entradas en cada diario), se filtró con el tópico energías renovables ${ }^{1}$. Esta búsqueda se realizó a través de los buscadores de cada uno de los diarios.

- Fase II. A la amplia muestra obtenida en la fase I, se le aplicaron los tres criterios siguientes, que se justifican de acuerdo con lo establecido en el currículo de ciencias español para la ESO en relación con la energía (véase más arriba), así como con las sugerencias de la literatura para un aprendizaje sobre esta desde una perspectiva CTS (García-Carmona y Criado 2008; Rose y Calabrese 2012; Solbes y Tarín 2004, etc.):

- Fase II.1 noticias que hacen referencia a la contribución y retos de la ciencia y/o tecnología a un futuro sostenible en términos energéticos;

- Fase II.2 noticias que muestran las ventajas y/o inconvenientes de las distintas fuentes de energía, sobre todo, desde el punto de vista medioambiental; y

- Fase II.3 noticias que muestran la influencia de factores sociales, políticos, económicos, culturales, etc. en asuntos energéticos.

De la fase II se obtuvo una muestra de 110 noticias. La búsqueda más intensa se hizo en la sección de ciencia y tecnología, pero no se descartaron otras secciones de los diarios, fundamentalmente en relación con el tercer criterio de esta segunda fase.

- Fase III. Finalmente, se hizo una selección más afinada buscando aquellas noticias que, a criterio de los autores de este trabajo (uno de ellos con amplia experiencia en el análisis y empleo de este recurso didáctico), presentaban de un modo más claro o explícito los criterios anteriormente señalados, teniendo en cuenta que estarían dirigidas a alumnado de ESO (12-16 años). De este último filtro surgió un repertorio final de 20 noticias relacionadas con la energía: 6 de $A B C, 7$ de El Mundo y 7 de El País.

\section{Resultados y discusión}

\section{Contenidos de las noticias de prensa relacionadas con la energía}

En la tabla 1 se muestra la información sobre los contenidos y las secciones de los diarios digitales donde aparecen publicadas las noticias seleccionadas. La temática más recurrente se refiere a las fuentes de energía (16 noticias), seguida del desarrollo científico y/o tecnológico en términos energéticos (10) y la política energética (8). Con menor frecuencia se tratan aspectos tales como el desarrollo sostenible (5) y la eficiencia energética (3).

\footnotetext{
${ }^{1}$ Este término se usó simplemente como palabra clave de búsqueda, pues sabemos que lo renovable o no renovable es la fuente que produce la energía.
} 
Tabla 1. Distribución de las noticias por temáticas y secciones de los diarios analizados.

\begin{tabular}{|l|l|c|c|c|}
\cline { 3 - 5 } \multicolumn{2}{l|}{} & ABC & El Mundo & El País \\
\hline Temáticas * & Fuentes de energía & $5 / 6$ & $5 / 7$ & $6 / 7$ \\
\hline & $\begin{array}{l}\text { Desarrollo científico y/o } \\
\text { tecnológico }\end{array}$ & $3 / 6$ & $3 / 7$ & $4 / 7$ \\
\hline & Política energética & $2 / 6$ & $3 / 7$ & $3 / 7$ \\
\hline & Desarrollo sostenible & $1 / 6$ & $2 / 7$ & $2 / 7$ \\
\hline \hline Secciones & Eficiencia energética & - & $2 / 7$ & $1 / 7$ \\
\hline & Medio ambiente & $1 / 6$ & $3 / 7$ & $3 / 7$ \\
\hline & Ciencia & $4 / 6$ & $5 / 7$ & $1 / 7$ \\
\hline & Tecnología & - & - & $3 / 7$ \\
\hline & Economía & $1 / 6$ & $1 / 7$ & $1 / 7$ \\
\hline & Política & - & - & $1 / 7$ \\
\hline & Opinión & - & - & - \\
\hline & Energía & Blog & $-1 / 7$ & - \\
\hline
\end{tabular}

Con respecto a las secciones donde aparecen las noticias relacionadas con la energía, puede decirse que prácticamente se encuentran en todas. En la muestra de noticias seleccionadas, predomina la sección de Ciencia, en la cual se encuentran la mitad de estas (10); el resto de las noticias se incluyen en las secciones de Tecnología (3), Economía (3), Política (1), Opinión (1), Energía (1) y Blog (1). Cabe destacar también que la mayoría de las noticias del diario El Mundo, incluidas en la sección de Ciencia, están escritas por los mismos autores (Teresa Guerrero y Miguel G. Corral; ambos periodistas científicos).

Por otro lado, en la tabla 2 se muestra la presencia de los tres criterios de selección establecidos para la búsqueda de las noticias en los tres diarios digitales. Como puede verse, la mayoría de las noticias alude a más de un criterio de los establecidos y, en todos los diarios, el criterio 2 (ventajas/inconvenientes de las distintas fuentes de energía) es el más frecuente. Asimismo, la mayoría de las noticias se refiere a la contribución de la ciencia y/o la tecnología a un futuro sostenible. El criterio que menor presencia tiene es el de la influencia de factores político-económicos en cuestiones energéticas (criterio 3).

Tabla 2. Distribución de los criterios a los que hacen referencia las noticias seleccionadas. ${ }^{2}$

\begin{tabular}{|l|c|c|c|c|}
\cline { 2 - 5 } \multicolumn{1}{c|}{} & ABC & El Mundo & El País & Total \\
\hline $\begin{array}{l}\text { Contribución y retos de la ciencia / tecnología a } \\
\text { un futuro sostenible en términos energéticos }\end{array}$ & $4 / 6$ & $4 / 7$ & $5 / 7$ & $13 / 20$ \\
\hline Ventajas / Inconvenientes de fuentes de energía & $6 / 6$ & $5 / 7$ & $6 / 7$ & $17 / 20$ \\
\hline $\begin{array}{l}\text { Influencia de factores político-económicos en } \\
\text { cuestiones energéticas }\end{array}$ & $2 / 6$ & $3 / 7$ & $3 / 7$ & $8 / 20$ \\
\hline
\end{tabular}

${ }^{2}$ La suma no es el número total de noticias porque los criterios no son excluyentes, sino que hay noticias que incluyen más de uno de ellos. 


\section{Descripción de las noticias y potencialidades didácticas para su uso escolar}

En este apartado se describe el contenido de las noticias seleccionadas, su potencial didáctico y algunas sugerencias para su implementación en clase de ciencias de la ESO. Ello se concretará, en el contexto de las distintas noticias, con una serie cuestiones abiertas y de carácter reflexivo que, en su conjunto, se orientan a desarrollar algunas de las dimensiones del pensamiento crítico (Blanco-López, España-Ramos y Franco-Mariscal 2017), tales como: entender la naturaleza compleja de la ciencia y la tecnología, así como sus relaciones con la sociedad; conocer temas científicos y socio-científicos, contrastando distintos puntos de vista; analizar críticamente la información; elaborar argumentos basados en información fiable; y desarrollar una opinión propia e independiente para la toma de decisiones.

\section{1. "Portugal ya cubre su demanda energética solo con renovables" (ABC, 2018)}

Informa que Portugal fue capaz de abastecerse energéticamente con energía procedente de fuentes renovables durante un periodo de tiempo, generando esperanza para un futuro sostenible. Se mencionan diferentes fuentes de energía renovables y los efectos medioambientales que tienen; por tanto, puede ser útil cuando se estén abordando las fuentes de energía; por ejemplo, pidiendo al alumnado que busque y/o amplíe información sobre las fuentes que se nombran. ${ }^{3}$ Asimismo, incide en que estas fuentes de energía son realmente viables para un futuro sostenible porque ya es posible usarla para obtener electricidad, sin apenas depender de las fuentes convencionales. Por otro lado, se menciona que la tecnología que usa Portugal es española y, además, que España recurre a Portugal cuando necesita energía; lo cual puede abrir un debate sobre por qué en España no ocurre lo mismo que en el país vecino en cuanto a la producción y consumo de energía procedentes de fuentes renovables. Podrían plantearse cuestiones como las siguientes:

- ¿Crees que sería posible que, en España, ocurriera lo mismo que en Portugal, es decir, que pudiéramos obtener toda la electricidad que necesitamos, sin usar fuentes de energía convencionales? Razona tu respuesta.

- ¿Piensas que tenemos alguna ventaja con respecto a Portugal para producir electricidad con fuentes de energía renovables? Arguméntalo.

- ¿Por qué crees que España no produce toda la electricidad que consume mediante fuentes de energía renovables?

\section{2. "La mayor planta solar térmica del mundo está en el desierto de Mojave" (ABC, 2014)}

Habla de una planta solar térmica en el estado de California, de su capacidad para producir energía y de cómo funciona; por tanto, es interesante para introducir la energía solar térmica. Explica que los espejos de la instalación están colocados en círculos concéntricos, lo cual sugiere plantear al alumnado:

- ¿Por qué crees que los espejos de las plantas solares térmicas se colocan en círculos concéntricos? Razona tu respuesta.

Asimismo, la noticia ayuda a ser consciente de las dimensiones de una planta de este tipo, en cuanto a la población a la que es capaz de abastecer, y la potencia energética que provee. La introducción de esta magnitud en este contexto es muy interesante porque el alumnado suele confundir energía con potencia; por lo que sería una buena ocasión para analizar su diferencia y relación mediante preguntas como:

\footnotetext{
${ }^{3}$ Un buen recurso, para ello, puede ser la página web de Red Eléctrica de España (ree.es/es). Ofrece información del consumo de energía eléctrica en España, en tiempo real, datos de la generación de esa energía con distintos tipos de fuentes, así como gráficos de evolución comparativa de tal generación con renovables y no renovables, en periodos de tiempo concretos.
} 
- ¿Qué unidad utiliza el autor de la noticia cuando babla de la capacidad de la central solar? ¿A qué magnitud corresponde esa unidad? ¿Cuál es la unidad de la energía eléctrica?

- ¿Qué diferencia crees que hay entre potencia y energía eléctrica?

Por otro lado, la noticia permite tratar los efectos de esta fuente de energía sobre el medio ambiente, tanto positivos (la reducción de emisiones de $\mathrm{CO}_{2}$ a la atmósfera) como negativos (impacto paisajístico). Se podría plantear en clase:

- El texto menciona que, a plena capacidad, esta central solar podría reducir la emisión de 400.000 toneladas de $\mathrm{CO}_{2}$ al año. Averigua qué cantidad de $\mathrm{CO}_{2}$ se emite al año en el estado de California y calcula si la reducción es grande o pequeña. Escribe un comentario reflexivo sobre esto.

3. "Físicos tratan de construir un «pequeño Sol» en la Tierra" (ABC, 2013)

Informa sobre la investigación llevada a cabo en la Universidad de Sevilla para conseguir una fuente de energía basada en la fusión nuclear. A nivel conceptual, permite abordar en clase la fusión nuclear:

- ¿Cuál es la estructura atómica del bidrógeno?

- ¿Qué son los isótopos y cuántos tiene el hidrógeno?

- ¿Qué característica puede presentar el bidrógeno para que sea el elemento que se usa en la fusión nuclear?

- ¿Por qué es necesario utilizar temperaturas tan altas para que se produzca la reacción?

- ¿Por qué para conseguir la fusión en la Tierra los reactores tienen que operar a temperaturas más elevadas que la del Sol? ¿Se te ocurre alguna explicación física?

Por otra parte, la noticia menciona las múltiples ventajas de esta fuente de energía, así como las dificultades para ponerla en marcha, mostrando los retos y límites a los que se enfrenta la comunidad científica al respecto. Ello favorece la reflexión sobre creencias desinformadas que suele tener el alumnado sobre la naturaleza de la ciencia, tales como que la construcción de conocimiento científico es inmediata; que los científicos trabajan de forma aislada, o que lo que los científicos investigan no influye en la sociedad. Se podría abordar con preguntas como:

- ¿Cuál es el problema que presenta la fusión nuclear?

- ¿A qué retos se enfrentan los científicos en este campo?

- ¿Qué importancia crees que tiene para esta línea de investigación que exista colaboración entre investigadores de distintas universidades?

Asimismo, sería interesante que el alumnado reflexionara sobre el título de la noticia:

- ¿Por qué crees que el autor de la noticia utiliza la expresión "pequeño Sol"? ¿Qué relación tiene con el texto?

4. "El sueño de la fusión nuclear, más cerca" (ABC, 2014)

Esta noticia también aborda la fusión nuclear y los esfuerzos de los científicos por conseguir de ella una fuente de energía limpia. Podrían trabajarse con ella prácticamente los mismos contenidos y preguntas que con la noticia anterior. Por tanto, sería interesante trabajarla junto con la anterior, pues son de años diferentes; lo cual permite mostrar al alumnado cómo la ciencia está en continuo avance, y los esfuerzos de los científicos de todo el mundo por lograr un futuro más sostenible. Tras la lectura de las dos noticias, se podría plantear al alumnado:

- ¿Qué diferencia(s) encuentras con respecto a la investigación científica de la fusión nuclear? ¿Hay avances? 
- ¿Están las investigaciones llevadas a cabo por los mismos cientificos? ¿Qué ha contribuido a avanzar en estas investigaciones?

- ¿Qué se deduce de toda esta información recopilada?

5. “¿Por qué estalló el reactor de la central nuclear de Chernóbil?" (ABC, 2016)

Está escrita por dos científicos expertos en Física Nuclear. Presenta cómo y por qué se produjeron los accidentes nucleares de Chernóbil y Fukushima. Una primera pregunta que se podría plantar al alumnado es:

- ¿Cuál es la principal diferencia en la causa de los accidentes nucleares de Chernóbily Fukushima?

Por otro lado, los autores defienden que, a pesar del rechazo que ha provocado estos accidentes hacia la energía nuclear en la sociedad, esta fuente de energía es una alternativa real y viable a los combustibles fósiles; y señalan que otras fuentes de energía renovables también pueden causar accidentes. Para reflexionar sobre todo esto, se podría preguntar:

- ¿Por qué estos cientificos consideran que la energía nuclear es una alternativa a los combustibles fósiles?

- ¿Qué ventajas y desventajas presenta la energía nuclear?

- ¿Cómo se gestionan los residuos que genera una central nuclear?

- ¿Por qué la radiación puede causar daños en el organismo?

- ¿Qué es más peligroso, el calentamiento global o la energía nuclear?

- ¿Qué opinión tienes, en general, sobre la energía nuclear?

6. "Victima de Chernóbil: "¿Qué vale más? ¿La vida, el futuro, nuestros hijos o una energía nuclear "barata"?)”" (ABC, 2016)

Expresa la opinión que tiene una de las víctimas del accidente nuclear de Chernóbil sobre la energía nuclear. Esta noticia permite introducir el tema de la energía nuclear complementando el contenido con otras fuentes (libros de texto, webs...) para que el alumnado compare y contraste la información de la noticia, y les ayude a comprender qué es o en qué consiste esta fuente de energía (qué es la radioactividad, sus posibles efectos nocivos en los seres humanos, etc.). También es idónea para reflexionar sobre el accidente nuclear que ocurrió en Chernóbil y sus consecuencias. Sería interesante tratarla en clase junto a la anterior noticia, y generar un debate en torno a las preguntas:

- ¿Te opondrías a la construcción de una nueva central nuclear en tu ciudad? ¿Por qué?

Además, la noticia indica la influencia de la política en este tema, haciendo así explícitas las conexiones de la ciencia con otros ámbitos como la sociedad o política y analizar sus interacciones:

- ¿Qué papel crees que deben tener la política y la sociedad, en general, en el debate sobre la energía nuclear? Argumenta tu respuesta.

Finalmente, podría ser interesante comparar esta con la anterior noticia para valorar las diferencias de opinión:

- ¿Qué diferencias presentan ambas noticias?

- ¿Cuál te parece más creíble o fiable, y por qué?

7. "El grafeno multiplica la cantidad de electricidad que se obtiene de la lus" (El Mundo, 2013)

Esta noticia informa del descubrimiento de un material que favorece la conversión de luz en electricidad. Por tanto, permite acercar a la sociedad los avances de la ciencia. Además, se mencionan la tecnología y las patentes, lo que permite abordar la diferencia entre ciencia y 
tecnología, pues en muchas ocasiones estos conceptos son considerados sinónimos o similares. Para ello, podría preguntarse al alumnado:

- ¿Qué es para ti la tecnología? ¿Y la ciencia?

- ¿En qué aspectos consideras que se parecen y diferencian?

Otro rasgo de la naturaleza de la ciencia que muestra esta noticia es que las investigaciones se llevan a cabo mediante colaboraciones entre científicos, incluso internacionales. La noticia da pie también al estudio de la corriente eléctrica (cómo se obtiene electricidad de la energía solar, cómo funcionan las células fotovoltaicas y cómo variaría con este nuevo material). Para enlazar la noticia con este estudio, podría preguntarse:

- El texto indica que el grafeno puede convertir la luz en electricidad, ¿cómo es posible?

- ¿Cómo se conoce a los dispositivos que transforman la luz en electricidad?

- ¿Qué material se suele usar en las células fotovoltaicas? ¿Por qué el grafeno es mejor?

También permite el análisis de las propiedades de este material e investigar su potencial en otras aplicaciones:

- ¿Conoces otras aplicaciones del grafeno? Si no es así, buiscalas.

- ¿Cuáles son las propiedades que lo hacen tan idóneo para esas aplicaciones?

Por otra parte, hace referencia a la necesidad de los países para apostar por la ciencia y cómo de gratificante es la profesión de científico, ya que se contribuye de alguna manera a una mejor sociedad. Para ello, podrían formularse las siguientes preguntas de reflexión:

- ¿Crees que los científicos trabajan solos y de manera aislada en sus laboratorios?

- ¿Cómo crees que es el trabajo de un cientifico? ¿Piensas que hacen siempre igual su trabajo? Coméntalo.

- ¿En qué grado consideras que la creatividad interviene en el desarrollo de la ciencia?

\section{8. "Las 'cracks' de las energías limpias" (El Mundo, 2014)}

La noticia se refiere a las investigaciones de cinco científicas sobre la producción de energía "limpia". Aborda la necesidad energética de la sociedad, el desconocimiento de muchas personas sobre la problemática energética, y la necesaria concienciación de la sociedad para hacer frente a estas situaciones. Luego, critica el apoyo que existe aún a los combustibles fósiles con la búsqueda de hidrocarburos en las Islas Canarias (España), o la puesta en marcha de proyectos de fracking para obtener gas. Al respecto, se podría preguntar al alumnado:

- ¿Por qué crees que se critica en el texto el uso de combustibles fósiles?

- ¿Piensas que se debería seguir buscando estas fuentes de energía? ¿Por qué?

Al hablar de las investigaciones de las cinco científicas, se hace referencia a alternativas energéticas existentes para alcanzar los objetivos de reducción de contaminantes, con vistas a un futuro sostenible. Entre estas investigaciones están la mejora de la producción de hidrógeno como alternativa al petróleo, y la eficiencia de las células solares. Por último, la noticia es interesante para analizar el papel de la mujer en la ciencia, tanto a lo largo de la historia como actualmente, porque critican expresamente los obstáculos que han encontrado en sus carreras científicas. Esto se puede reforzar en clase con preguntas como:

- ¿Conoces mujeres cientificas del pasado? ¿A cuántas? ¿Y a cuántos hombres cientificos del pasado?

- ¿Crees que no se han conocido mujeres cientificas porque no las habia, o porque sus investigaciones no eran importantes?

- ¿Qué motivos encuentras para que se considerara en el pasado que la ciencia era cosa de hombres?

- ¿Crees que actualmente las mujeres tienen problemas para acceder a la ciencia? ¿Por qué? 
- ¿Qué obstáculos mencionan las cientificas del texto que tienen las mujeres en la carrera cientifica? ¿Se da este problema en otras profesiones?

- ¿Debería la sociedad ser más consciente y tomar medidas? ¿Qué tipo de medidas se te ocurren?

9. “¿Por qué puede el fracking causar terremotos?” (El Mundo, 2015)

Artículo de opinión escrito por un físico cuya investigación se centra en la Física del Clima y de la Atmósfera de la Tierra. Explica la técnica de fracking de una forma muy comprensible. Lo explica mediante una analogía con una esponja, que podría realizarse en el laboratorio escolar a partir de la lectura del artículo. También aborda el tema de la necesidad energética, pero critica que se sigan buscando combustibles fósiles y que no se apueste por fuentes de energía limpias, atribuyendo la principal responsabilidad al dinero. De este modo, incide en la influencia de los factores económicos sobre los medioambientales. Al hilo de esto, sería interesante preguntar al alumnado:

- ¿Te parece que esta técnica aporta algo al problema del agotamiento de los combustibles fósiles? ¿Por qué?

- Aun conociendo los posibles riesgos del fracking, żuál es tu opinión acerca de su utilización como método de obtención de energía?

La noticia también invita a una reflexión sobre la situación energética que vivimos y las graves consecuencias que tendremos, si todo continúa igual, pues el propio autor termina con una serie de preguntas para reflexionar: "¿No deberíamos buscar otras soluciones a los problemas que nos asaltan, soluciones distintas de seguir haciendo lo mismo que hemos hecho en el pasado?”, "¿Cambiamos?”.

\section{0. "Hacia el país 100\% renovable" (El Mundo, 2016)}

Informa de la capacidad de Costa Rica para abastecer su demanda de electricidad durante 76 días con fuentes de energías limpias. Hace referencia a las distintas fuentes de energía renovables y a los beneficios medioambientales que éstas tienen. Asimismo, muestra que este tipo de fuentes son realmente viables como alternativa para un futuro sostenible. Pone de manifiesto el caso de otros países que también cubren parte de su demanda energética con energías limpias, y cómo es necesario aprovechar los recursos que ofrece la naturaleza para la sostenibilidad. Podrían plantearse las siguientes cuestiones al alumnado:

- ¿Qué ventajas crees que tiene España respecto a otros países en cuanto a recursos de la naturaleza?

- ¿Crees que está aprovechando estos recursos para obtener energía? ¿Por qué?

Además, esta noticia habla de los retos que la ciencia tiene aún para conseguir un mejor aprovechamiento de estas energías; sobre todo de la solar y eólica. Por otro lado, la noticia incluye gráficos de producción eléctrica y la evolución de la generación eléctrica de Costa Rica en los últimos años; lo cual puede servir para trabajar con el alumnado la interpretación de datos en un contexto concreto. También podrían trabajarse los conceptos de energía y potencia a partir del planteamiento siguiente:

- El texto expone que tenemos 106.000 MW de potencia instalada y que la demanda punta del año 2015 fueron $40.000 \mathrm{MW}$. ¿Qué significa esto? ¿Qué magnitud utiliza?

- ¿Qué es la potencia? ¿Es lo mismo que la energía? ¿Qué unidades tiene la energia? ¿Qué diferencia hay entonces entre la energía y la potencia?

\section{1. "El carbón mata 20 veces más gente que Chernóbil" (El Mundo, 2016)}

Recoge la entrevista a un científico muy implicado en los retos energéticos a los que se enfrenta la Humanidad. Habla de la problemática medioambiental a la que nos enfrentamos: cambio climático y contaminación ambiental, debido principalmente a la quema de 
combustibles fósiles. Hace una crítica a estas fuentes de energía, aunque se muestra escéptico con las renovables, defendiendo la energía nuclear de fisión como la solución. También menciona la fusión nuclear, si bien tampoco considera que pueda ser la solución en un futuro debido a factores económicos. Por ello, esta noticia permite conocer y aprender sobre las diferentes fuentes de energía, así como las ventajas e inconvenientes de cada una de ellas. También permite abordar los retos de la ciencia y de la tecnología en cuanto a la búsqueda de técnicas que sean capaces de capturar los contaminantes que han sido emitidos. Por otro lado, hace una crítica a la insensatez de los gobiernos de seguir apoyando económicamente los combustibles fósiles: "[...] se subsidia el uso de combustibles fósiles por todo el mundo. La Agencia Internacional de la Energía cree que se destinan 550.000 millones de dólares cada año para subsidios directos para el uso de estas fuentes fósiles [...]. Y esa cantidad es cuatro veces mayor que los subsidios para las energías renovables. No tiene sentido."

En general, es una noticia que permite no solo aprender sobre las distintas fuentes de energía y reflexionar sobre la problemática energética que vivimos, sino también analizar y comprender los factores que influyen y que llevan a dicha problemática. Para ello, tras la lectura de esta noticia podrían plantearse preguntas como:

- ¿Qué soluciones crees que existen ante la problemática energética?

- ¿Qué responsabilidad crees que tiene los gobiernos en esta problemática?

- ¿Por qué crees que no se dan más ayudas a las fuentes de energías renovables?

- ¿Qué soluciones propondrías tú?

\section{2. "Un mundo de energía 100\% limpia es posible" (El Mundo, 2016)}

La noticia muestra la entrevista a un investigador estadounidense dedicado a cuestiones ambientales y energéticas. El investigador indica que un mundo con fuentes de energía 100\% renovables es posible en un futuro, mostrando sus múltiples ventajas. El interés didáctico de esta noticia es que permite aprender sobre fuentes de energía, sus consecuencias en el medioambiente y en la sociedad, así como sobre los avances de la tecnología para conseguir un futuro sostenible. También indica cómo ha llevado a cabo su investigación, poniendo así de manifiesto que no existe un único método científico. Para reflexionar sobre esto, se podría preguntar al alumnado:

- ¿Cómo ha llevado este cientifico su investigación?

- ¿Creéis que todos los científicos siguen este método de investigación? Coméntalo.

- ¿Conocéis otros métodos? ¿Cuáles?

Asimismo, el entrevistado indica que "se reducirá la necesidad de importar energía, y los conflictos internacionales por fuentes de energía." Por tanto, permite debatir y reflexionar sobre los problemas económicos y políticos derivados de la producción y consumo de la energía no renovables. Sería preguntas propicias para ello las siguientes:

- ¿Qué problemas provocan las fuentes de energía convencionales, además de los medioambientales?

- ¿Por qué crees que el entrevistado afirma que las fuentes de energía convencionales provocan problemas políticos y económicos?

13. "El futuro de la energía" (El Mundo, 2016)

Artículo de opinión donde el autor reflexiona sobre la problemática de la energía y su futuro. En este sentido, tras su lectura se podría empezar preguntando al alumnado:

- ¿Cuál crees que es la intención del autor al escribir este artículo?

- ¿Crees que se trata de un texto objetivo o subjetivo? Coméntalo.

- ¿Qué comportamiento crees que debería adoptar un lector cuando lee un texto de este tipo? 
Asimismo, permite resaltar la importancia de la energía en la sociedad y la economía de los países, y de su consumo como indicador de desarrollo socioeconómico. Por otro lado, en la noticia se hacen afirmaciones como: "Así, por ejemplo, la visión del petróleo como un emisor masivo de CO2 no se ve avalada por los datos en contradicción con la percepción que, sobre este asunto, tiene el conjunto de la opinión pública [...]"; la cual indica que la información que aparece en la prensa debe ser analizada críticamente, y no debe tomarse como verdades absolutas; especialmente, en lo que se refiere a temas científicos. Para poner al alumnado a reflexionar sobre el contenido del texto, se puede preguntar:

- ¿Qué posición tiene el autor respecto a las fuentes de energía convencionales? ¿Qué argumentos da?

- ¿Estás de acuerdo con lo que afirma? ¿Por qué?

- ¿Crees que toda la información cientifica que expone es correcta? Explicalo.

- ¿Qué argumentos usarías para oponerte a los del autor?

\section{4. "El hidrógeno llama a la puerta" (El País, 2018)}

Noticia que informa del desarrollo científico para conseguir que el hidrógeno sea una fuente de energía renovable. Permite aprender sobre distintas fuentes de energías no renovables y sus inconvenientes, así como las características del hidrógeno, que lo convierten en un candidato ideal para usarlo como fuente de energía. Como preguntas explícitas para que el alumnado piense sobre ello, podrían plantearse las siguientes:

- ¿Por qué el hidrógeno es noticia?

- ¿Qué características presenta el hidrógeno para que sea un elemento idóneo para producir energía?

- ¿Crees que se eliminarían todos los problemas medioambientales usando bidrógeno? Razona tu respuesta.

La noticia explica también los mecanismos de obtención del hidrógeno, lo cual permitiría abordar esta fuente de una forma motivadora para el alumnado. Para ello, se podría plantear:

- ¿De qué manera se obtiene el hidrógeno según el texto?

- ¿Sabes en qué consisten estos métodos? Busca en el libro de texto u otras fuentes esta información.

Igualmente destaca el desarrollo llevado a cabo por empresas para obtenerlo usando energía eólica o solar, posibilitando así enlazar con el estudio de estas fuentes. Una pregunta para propiciar esto podría ser:

\section{- ¿Cómo es posible obtener el hidrógeno a partir de fuentes de energía renovables?}

Además, la noticia indica las aplicaciones que puede tener esta fuente, mostrando las ventajas que proporcionaría para un futuro sostenible energéticamente.

\section{5. "Un vuelo de esperanzas para las energías renovables" (El País, 2016)}

Esta noticia expone el viaje que ha realizado un avión movido exclusivamente por energía solar. Por un lado, permite aprender sobre la energía solar y su potencial como fuente de energía renovable, en aras de cambiar la situación grave a la que se enfrenta la Humanidad, derivada del consumo de energía (agotamiento de los recursos, contaminación atmosférica y efecto invernadero). Por otro, contribuye a tomar conciencia de que las fuentes de energía renovables son una alternativa real y viable, puesto que ha permitido realizar un vuelo. Asimismo, la noticia hace afirmaciones con contenido científico, como que el vuelo se ha realizado: "[...] intercambiando de manera autónoma casi 2.500 kilovatios por hora entre el sol, las baterias y sus motores [...]". Afirmaciones de este tipo pueden conducir al alumnado a activar el conocimiento científico para poder comprender lo que se está leyendo; por ejemplo, con preguntas como la siguiente: 
- ¿Qué significa esta afirmación?

- ¿Qué unidades utiliza? ¿A qué magnitud física se refiere?

Por último, la noticia también indica que: "La tecnología tiene que aportar los medios y mecanismos que hagan posible un desarrollo económico y social compatible con los recursos del planeta". Esto da pie a trabajar las diferencias y similitudes entre en ciencia y tecnología. Para ello, se puede preguntar:

- ¿Estás de acuerdo con esta afirmación? Explícalo.

- ¿Qué rasgos caracterizan a la tecnología? ¿Y a la ciencia?

- ¿Qué diferencias y similitudes encuentras entre ciencia y tecnología?

16. "Cuatro formas sorprendentes de producir energía" (El País, 2016)

Trata sobre inventos tecnológicos para producir energía de una forma original e innovadora. Pone de manifiesto que es necesario buscar alternativas para conseguir un futuro energético sostenible, y cómo la creatividad es un factor clave en ello. Además, a nivel conceptual ayuda a entender qué es la energía y qué tipos de energía pueden distinguirse, así como la ciencia que hay detrás de cada forma de producir energía. Igualmente permite analizar las ventajas e inconvenientes de las distintas formas de obtener energía que se describen. Por otro lado, esta noticia pone de manifiesto que la energía es un indicador de desarrollo socioeconómico. Para llevar poner al alumnado a reflexionar sobre estos aspectos, se podrían hacer las siguientes preguntas:

- ¿Qué han llevado a los investigadores a proponer estas formas de producir energía?

- ¿Cuál te parece más original y por qué?

- ¿Crees que en la ciencia interviene la creatividad? Explicalo.

- ¿Crees que los gobiernos deberían tener en cuenta estas formas de producir energía? Razona tu respuesta.

\section{7. "El petróleo de la discordia" (El País, 2014)}

Esta noticia se encuentra en la sección de Política, e incluye entrevistas a diferentes personas de diferentes ámbitos (científico, económico, agrícola, construcción, entre otros) sobre la búsqueda de petróleo llevada a cabo en las Islas Canarias (España). Puede ayudar al alumnado a aprender sobre el petróleo, su procedimiento de extracción, etc., además de las consecuencias que ello tiene para la sociedad, la economía y el medio ambiente. Asimismo, pone de relieve la influencia de la política en cuestiones energéticas. Por otra parte, permite conocer y analizar la opinión de diferentes personas, conociendo los argumentos y motivos que utilizan para defender sus posturas. Una reflexión sobre todo ello se podría propiciar en el aula con cuestiones como las siguientes:

- ¿Estás de acuerdo con alguna opinión de las expuestas? ¿Por qué?

- ¿En qué medida te convencen algunos de los argumentos de los que no comparten tu opinión? ¿Crees que existe alguna opinión más válida que otra? Explica tus motivos.

- ¿Tienen todos los políticos la misma opinión? ¿Cuál es la tuya?

18. "Un año menos para el fin del mundo" (El País, 2017)

Esta noticia aborda la problemática del calentamiento global debido a la actividad humana. Su lectura puede ayudar a que el alumnado tome conciencia de las terribles consecuencias que trae consigo tal fenómeno. Hace referencia también a distintas fuentes de energía, y a sus ventajas e inconvenientes, lo que permite aprender sobre ellas en este contexto. Además, enfatiza la importancia de la ciencia y la innovación tecnológica para mejorar el conocimiento y capacidad de las distintas fuentes de energía. Expone la necesidad de tomar medidas políticas 
que afronten y reviertan esta situación, mediante una transición energética que apueste por fuentes de energía sostenibles. Asimismo, la noticia apela a la responsabilidad de la ciudadanía al respecto, a la que critica por su baja implicación. Para reflexionar sobre ello, en el contexto de la noticia, se podrían plantear las siguientes cuestiones al alumnado:

- ¿Crees que la sociedad es consciente del problema energético que tenemos? Coméntalo.

- ¿Crees que debería informarse más a los ciudadanos? ¿Por qué?

- ¿Crees que como sociedad podemos hacer algo al respecto o no es necesario? Coméntalo.

- ¿Qué se te ocurre que podríamos hacer como ciudadanía para contribuir a un mundo sostenible?

- ¿Crees que los gobiernos se toman en serio el problema? Coméntalo.

- ¿Qué papel deberían tener, en tu opinión, la ciencia y la innovación ante el problema?

19. "Sol sin sol" (El País, 2015)

Esta noticia de opinión critica que, en un país como España, donde hay muchas horas de Sol, no se apueste por la energía solar. Permite, pues, poner a pensar al alumnado sobre las ventajas de la energía solar, y sobre la influencia que tiene la política, dominada por la economía, sobre cuestiones energéticas, tales como las trabas legislativas y la existencia del conocido "impuesto al sol". Para que el alumnado reflexione sobre ello, en el contexto de la noticia, se podrían plantear las siguientes cuestiones:

- ¿Por qué crees que, en España, no se apostaba por la energía solar, en el momento en que fue publicada esta noticia? Analiza todos los factores que piensas que pudieron influir en ello.

- ¿Te parece adecuada la postura que adoptó ese gobierno? ¿Por qué?

- ¿Qué argumentos usarías para convencer a otras personas de que el conocido como "impuesto al sol" no es una buena idea, desde un punto de vista energético, económico y medioambiental?

20. "Las plantas solares también pueden flotar" (El Pais, 2017)

Esta noticia informa sobre plantas solares que se están empezando a instalar en el mar. Permite aprender sobre las fuentes de energías renovables, sus ventajas, en general, y su capacidad para producir energía, en particular. Asimismo, hace referencia a la aportación de la ciencia y la tecnología para seguir avanzando en materia energética de forma sostenible. Esto pone de manifiesto también el carácter dinámico y evolutivo de la ciencia. Se menciona también que una de esas aportaciones de la tecnología es el desarrollo de las plantas solares marinas. Esto se puede utilizar para reflexionar y aprender sobre las ventajas e inconvenientes que presentan tales plantas respecto a las plantas solares convencionales: eficiencia, durabilidad de los materiales con las que están fabricadas, etc. Esto se puede incitar con preguntas del tipo:

- ¿Crees que es una buena idea las plantas solares marinas? Razona tu respuesta.

- ¿Te parecen más adecuadas que las terrestres? ¿Por qué?

- ¿Cuál es el motivo que ha llevado a los científicos a plantear el desarrollo de estas plantas solares?

- ¿Por qué crees que no se planteó antes el desarrollo de las plantas solares marinas?

\section{Conclusión}

La alfabetización científica que demanda nuestra sociedad pasa necesariamente por mejorar no solo el conocimiento científico de la ciudadanía, sino también sus habilidades para la reflexión y argumentación crítica. Ello requiere, entre otros aspectos, el desarrollo de la competencia para leer, comprender y analizar noticias de carácter científico-tecnológico. Los medios de comunicación y, en especial la prensa digital, juegan un papel esencial en el fomento de esa alfabetización científica. Y su utilización en las aulas puede propiciar situaciones de aprendizaje en torno a problemáticas reales; lo cual supone la apertura de la educación 
científica al ámbito no formal, favoreciendo de este modo una perspectiva más utilitaria de la enseñanza de las ciencias.

Tras el análisis presentado de noticias de la prensa digital diaria sobre temas relacionados con la energía, podemos decir que estos reciben una atención notoria en los principales diarios españoles. Cuestiones como el cambio climático, el agotamiento de recursos energéticos, el uso de fuentes de energía renovables vs. no renovables, y el impacto que todo ello puede tener en la calidad de vida de la sociedad, entre otras, forman parte de nuestro día a día. La variedad de noticias y las diferentes secciones de los diarios en las que estas aparecen, denota que los asuntos energéticos constituyen un problema complejo que no es solo científico-tecnológico, sino también social, político y económico, que afecta a distintos actores de la sociedad; esto es, con una marcada dimensión CTS.

Con respecto al potencial didáctico de las noticias analizadas ${ }^{4}$, puede decirse que todas presentan aspectos que las hacen idóneas para usarlas en clase de ciencias; especialmente las que hacen referencia a las fuentes de energía (tipos, ventajas e inconvenientes de cada una, impacto socioambiental, etc.). Permiten abordar reflexivamente la problemática energética actual y venidera, en aras de vislumbrar un futuro energético sostenible y determinar qué responsabilidad debería tener la ciudadanía en ello. Asimismo, las noticias favorecen la discusión sobre determinados aspectos de la naturaleza de la ciencia y la tecnología, tales como la idea de desarrollo y dinamismo permanente de estas; el carácter colaborativo entre los profesionales de este ámbito en sus investigaciones; la diversidad metodológica en la investigación científica; o el papel de las científicas en el desarrollo de la ciencia y los obstáculos que encuentran por su condición de mujeres. Además, ciertas noticias son idóneas para tratar algunas de las ideas alternativas que, con frecuencia, muestra el alumnado con respecto al concepto de energía, tales como la confusión entre energía y potencia, o entre energía y combustible.

Por todo ello, consideramos que promover la reflexión sobre cuestiones CTS relativas a la energía, mediante noticias de la prensa digital, puede favorecer dos aspectos didácticos esenciales: (1) la contextualización de la ciencia escolar en la realidad cotidiana, mostrando así al alumnado que los contenidos que se trabajan en clase tienen presencia en su día a día y en la sociedad, en general; y (2) el desarrollo del pensamiento crítico en el alumnado ante un tema socio-científico controvertido, con vistas a construir ideas y opiniones propias para la adopción de decisiones responsables. En definitiva, se espera que la muestra de noticias presentada y las orientaciones dadas para su posible implementación en el aula, sirvan de referente o recurso a otro profesorado interesando en tratar la energía desde una perspectiva CTS.

\section{Referencias bibliográficas}

Acevedo, J.A. (2009) Cambiando la práctica docente en la enseñanza de las ciencias a través de CTS. En M. Martín Gordillo (coord.), Educación, Ciencia, Tecnología y Sociedad (pp. 35-40). Madrid: OEI.

Alfaro López, H.G. (2010) La lectura como proceso de comprensión y conocimiento científico. Investigación Bibliotecológica 24 (50), 35-47.

Aragón, M.M. (2004) La ciencia de lo cotidiano. Revista Eureka sobre Enseñanza y Divulgación de las Ciencias 1 (2), 109-121.

Bennett, J. Lubben, F., Hogarth, S. (2007) Bringing science to life: A synthesis of the research evidence on the effects of context-based and STS approaches to science teaching. Science Education 91 (3), 347-370.

\footnotetext{
4 Todas las noticias seleccionadas están accesibles en abierto online; basta con introducir el título en un buscador
} de Internet para localizarlas. 
Blanco-López, A., España-Ramos, E., Franco-Mariscal, A.J. (2017) Estrategias didácticas para el desarrollo del pensamiento crítico en el aula de ciencias. Ápice. Revista de Educación Científica 1(1), 107-115.

Demeo, A.E., Feldman, D.P., Peterson, M.L. (2013) A human ecological approach to energy literacy through hands-on projects: An essential component of effectively addressing climate change. Journal of Sustainability Education 4, 1-16.

Doménech, J.L., Gil-Pérez, D., Gras-Martí, A., Guisasola, J., Martínez-Torregrosa, J., Salinas, J., ... Vilches, A. (2007) Teaching of energy issues: A debate proposal for a global reorientation. Science \& Education 16 (1), 43-64.

Ezquerra, A., Fernández, B. (2014) Análisis del contenido científico de la publicidad en la prensa escrita. Revista Eureka sobre Enseñanza y Divulgación de las Ciencias 11 (3), 275-289.

FECYT [Fundación Española para la Ciencia y la Tecnología] (2018) Principales resultados de la encuesta de percepción social de la ciencia 2018. Disponible en: https://www.fecyt.es/es/noticia/principales-resultados-de-la-encuesta-de-percepcionsocial-de-la-ciencia-2018

Fleming, R. (1986) Adolescent reasoning in socio-scientific issues, part I: Social cognition. Journal of Research in Science Teaching 23 (8), 677-687.

García-Carmona, A. (2005) Relaciones CTS en el estudio de la contaminación atmosférica: Una experiencia con estudiantes de Secundaria. Revista Electrónica de Enseñanza de las Ciencias 4 (2), 1-17.

García-Carmona, A. (2006) Interacciones CTS en el aprendizaje del electromagnetismo: Una experiencia para el desarrollo de actitudes de responsabilidad. Investigación en la Escuela 58, 79-92.

García-Carmona, A. (2008) Relaciones CTS en la educación científica básica II: Investigando los problemas del mundo. Enseñanza de las Ciencias 26 (3), 389-402.

García-Carmona, A. (2014) Naturaleza de la ciencia en noticias científicas de la prensa: análisis del contenido y potencialidades didácticas. Enseñanza de las Ciencias 32 (3), 493-509.

García-Carmona, A. (2015) Noticias sobre temas de Astronomía en los diarios: un recurso para aprender sobre la naturaleza de la ciencia reflexivamente. Revista de Enseñanza de la Física 27 (1), 19-30.

García-Carmona, A., Acevedo, J.A. (2016) Learning about the nature of science using newspaper articles with scientific content. Science \& Education 25 (5-6), 523-546.

García-Carmona, A., Criado, A.M. (2008) Enfoque CTS en la enseñanza de la energía nuclear: Análisis de su tratamiento en textos de Física y Química de la ESO. Enseñanza de las Ciencias 26 (1), 107-124.

García-Carmona, A., Criado, A.M. (2010) La competencia social y ciudadana desde la educación científica: Una experiencia en torno a la energía nuclear. Investigación en la Escuela 71, 25-38.

Gómez Romero, P. (2007) Un planeta en busca de energía. Madrid: Síntesis.

Hazelkorn, E., Ryan, C., Beernaert, Y., Constantinou, C.P., Deca, L., Grangeat, M., ... WelzelBreuer, M. (2015) Science education for responsible citizenship. Brussels: European Commission.

Hobbs, R., Jensen, A. (2009) The past, present, and future of media literacy education. Journal of Media Literacy Education 1 (1), 1-11. 
Hodson, D. (2003) Time for action: Science education for an alternative future. International Journal of Science Education 25 (6), 645-670.

Hodson, D. (2008) Towards scientific literacy. Rotterdam: Sense Publishers.

Jarman, R., McClune, B. (2007) Developing scientific literacy. Using the news media in the classrom. New York, NY: Open University Press.

Jiménez-Liso, M.R., Hernández-Villalobos, L., Lapetina, J. (2010) Dificultades y propuestas para utilizar las noticias científicas de la prensa en el aula de ciencias. Revista Eureka sobre Enseñanza y Divulgación de las Ciencias 7(1), 107-126.

Mejlgaard, N., Bloch, C., Degn, L., Nielsen, M. W., Ravn, T. (2012) Locating science in society across Europe: Clusters and consequences. Science and Public Policy 39 (6), 741-750.

Miller, J. D. (2004) Public understanding of, and attitudes toward, scientific research: What we know and what we need to know. Public Understanding of Science 13 (3), 273-294.

Real Decreto 1105/2014, de 26 de diciembre, por el que se establece el currículo básico de la Educación Secundaria Obligatoria y del Bachillerato. Boletín Oficial del Estado. Madrid, 3 de enero de 2015, núm. 3, pp. 169-546.

Nordine, J., Krajcik, J., Fortus, D. (2011) Transforming energy instruction in middle school to support integrated understanding and future learning. Science Education 95 (4), 670-699.

Oliveras, B., Sanmartí, N. (2009) La lectura como medio para desarrollar el pensamiento crítico. Educación Química 20 (1), 233-245.

Oliveras, B., Márquez, C., Sanmartí, N. (2013) The use of newspaper articles as a tool to develop critical thinking in science classes. International Journal of Science Education 35 (6), 885-905.

Rocard, M., Csermely, P., Jorde, D., Lenzen, D., Walberg-Henriksson, H., Hemmo, V. (2007) Science education now. A renewed pedagogy for the future of Europe. Brussels: European Comission.

Rose, S.L., Calabrese, A. (2012) Should great lakes city build a new power plant? How youth navigate socioscientific issues. Journal of Research in Science Teaching 49 (5), 541-567.

Solbes J. (2011) ¿Por qué disminuye el alumnado de ciencias? Alambique 67, 53-61.

Solbes, J., Tarín, F. (2004) La conservación de la energía: un principio de toda la física. Una propuesta y unos resultados. Enseñanza de las Ciencias 22 (2), 185-194.

Yuenyong, C., Jones, A., Sung-Ong, S., Yutakom, N., Forret, M. (2006) Teaching and learning about energy: Using Science, Technology and Society (STS) approach. Bangkok: Kasetsart University. 